\title{
WELFARE MEASUREMENT AND MEASUREMENT ERROR
}

\author{
Andrew Chesher \\ Christian Schluter
}

THE INSTITUTE FOR FISCAL STUDIES DEPARTMENT OF ECONOMICS, UCL cemmap working paper CWP03/01 


\title{
Welfare Measurement and Measurement Error
}

\author{
ANDREW CHESHER* \\ UNIVERSITY COLLEGE LONDON \\ Christian Schluter ${ }^{\dagger}$ \\ University of BRISTOL AND CASE LSE
}

April 2nd 2001

\begin{abstract}
The approximate effects of measurement error on a variety of measures of inequality and poverty are derived. They are shown to depend on the measurement error variance and functionals of the error contaminated income distribution, but not on the form of the measurement error distribution, and to be accurate within a rich class of error free income distributions and measurement error distributions. The functionals of the error contaminated income distribution that approximate the measurement error induced distortions can be estimated. So it is possible to investigate the sensitivity of welfare measures to alternative amounts of measurement error and, when an estimate of the measurement error variance is available, to calculate corrected welfare measures. The methods are illustrated in an application using Indonesian household expenditure data.
\end{abstract}

KEY WoRDS: inequality measurement, poverty indices, measurement error.

JEL Classification: D31, I32, C14

${ }^{*}$ Corresponding address: Department of Economics, University College London, Gower Street, London WC1E 6BT, U.K. andrew.chesher@ucl.ac.uk.

${ }_{\dagger}^{\dagger}$ Email: c.schluter@bristol.ac.uk. 


\section{INTRODUCTION}

Most of the attention paid to statistical aspects of inequality and poverty measurement has focussed on properties of the sampling distributions of inequality statistics (see Davidson and Duclos (1997)), regarding the data employed to calculate the statistics as error free observations $^{1}$. Of course data are rarely, if ever, error free. The tension between the desire for large samples and accurate measurement always results in measurement error being present.

Measurement of income inequality and poverty is essentially measurement of variability. When measurement error is not strongly negatively correlated with income, recorded income is more variable than actual income causing calculated inequality measures to tend to overstate true inequality. Improvements in the quality of survey instruments reduce measurement error and can lead to apparent reductions in inequality when true inequality may be unchanged or increased. Comparisons across groups of the population, for example urban and rural dwellers, are affected by differences in the extent of measurement error within groups. The result is that measurement error can cause targeted redistributional policies to be aimed incorrectly and can lead to false conclusions concerning the impact of poverty alleviating measures. Measurement error affects some inequality measures more than others and the effect of measurement error depends upon the distribution of true income. This paper investigates the differential effect of measurement error on a variety of inequality measures. It studies the relationship between, on the one hand, the impact of measurement error on inequality and poverty measures and on the other, the form of the underlying structural income distribution.

To obtain the precise effects of measurement error on inequality measures requires a complete specification of the joint distribution of income and measurement error and exact results require a case by case approach from which it is difficult to draw general conclusions. This paper addresses this problem by considering the approximate effects of measurement error. These are shown to depend upon particular features of the true income distribution, the features that are relevant being determined by the inequality measure under consideration. The only feature of the measurement error distribution appearing in the approximation is its variance, measured in an appropriate metric. The approximate impact of measurement error can be estimated for any candidate value of the measurement error variance using only error contaminated income data. This allows investigation of the sensitivity of computed inequality and poverty measures to measurement error.

Although this paper focuses on the effect of measurement error on welfare measurement, its results can be applied to other problems with a similar structure. For example, income reported over a fixed period and "permanent" income may be related to one another in the same way as the error contaminated and error free income discussed in this paper. For such cases the results of this paper shed light on the relationship between

\footnotetext{
${ }^{1}$ There are a few exceptions. Cowell and Victoria-Feser (1996) examine the statistical robustness to outliers of a variety of inequality indices. Analysis of measurement error effects using fully parametric approaches and specific income and measurement error distributions are set out in Israelsen, McDonald and Newey (1984) and van Praag, Hagenaars and van Eck (1983). See also Ravallion (1994).
} 
welfare measures calculated using survey records of income over reporting periods of different lengths and welfare measures calculated using "permanent" income. Of course real short run variation in income may have substantive welfare implications, unlike the spurious measurement error which motivates this paper. Although the exposition is cast in terms of household or personal income, the results given here apply when household expenditures are the basis for calculating welfare measures, and they apply in other similar situations, for example in the measurement of industrial concentration or of inequality in the distribution of time spent unemployed.

We consider welfare measures, specifically income inequality measures and poverty indices, which can be expressed as a functional, $\mathcal{W}\left(F_{Y}\right)$, of the distribution function, $F_{Y}$, of income, $Y$. With error free income denoted by $X$ and error contaminated income denoted by $Z$, we study the relationship between $\mathcal{W}\left(F_{Z}\right)$ and $\mathcal{W}\left(F_{X}\right)$ and show that the error contaminated measure, $\mathcal{W}\left(F_{Z}\right)$, is approximately equal to

$$
\mathcal{W}^{A}\left(F_{X}, \sigma^{2}\right)=\mathcal{W}\left(F_{X}\right)+\sigma^{2} \mathcal{W}^{*}\left(F_{X}\right)
$$

where $\mathcal{W}^{*}$ is a functional specific to each welfare measure and independent of the measurement error distribution, and $\sigma^{2}$ is the variance of measurement error expressed in an appropriate metric. We derive the form of $\mathcal{W}^{*}$ for a rich class of welfare measures.

In the approximation, $\mathcal{W}^{A}\left(F_{X}, \sigma^{2}\right)$, the term $\mathcal{W}^{*}\left(F_{X}\right)$ can be replaced by $\mathcal{W}^{*}\left(F_{Z}\right)$ without disturbing the order of the approximation error. Survey data regarded as realisations of $Z$ can be used to produce estimates, $\mathcal{W}\left(\hat{F}_{Z}\right)$ and $\mathcal{W}^{*}\left(\hat{F}_{Z}\right)$, leading to measurement error corrected estimates of welfare measures, $\mathcal{W}\left(\hat{F}_{Z}\right)-\hat{\sigma}^{2} \mathcal{W}^{*}\left(\hat{F}_{Z}\right)$ where $\hat{\sigma}^{2}$ is either an extraneous estimate of the measurement error variance, or, in a sensitivity analysis, a candidate value from a plausible range of values.

Many welfare measures and all those considered here, can be expressed in terms of the particular functional

$$
\mathcal{W}\left(F_{Y}\right)=\mathcal{L}\left(a, N(y, a) ; F_{Y}\right)=\int_{0}^{a} N(y, a) d F_{Y}(y)
$$

for some function of income $N(y, a)$ and limit of integration $a$. Table 1 shows the measures examined in this paper, defined in terms of $\mathcal{L}$. A comprehensive survey of the literature on inequality and poverty measurement is given in respectively Cowell (2000) and Seidl (1988).

The mean, $E[Y]$, and $p$-quantile, $Q_{Y}(p)$, which appear as components of some of these measures are, in terms of the functional $\mathcal{L}$, as follows

$$
\begin{aligned}
E(Y) & =\mathcal{L}\left(\infty, y ; F_{Y}\right) \\
p & =\mathcal{L}\left(Q_{Y}(p), 1 ; F_{Y}\right)
\end{aligned}
$$

the $p$-quantile being implicitly defined by the second of these equations.

Table 2 shows the approximation, $\mathcal{W}^{A}\left(F_{X} \cdot \sigma^{2}\right)$, for each error contaminated welfare measure, $\mathcal{W}\left(F_{Z}\right)$, that we consider. In each case functionals of $F_{X}$ can be replaced by the same functionals of $F_{Z}$ without changing the order of the approximation error, and these are readily estimated. For example, the difference between the error contaminated and error free poverty gap index is $\frac{\sigma^{2}}{2} c f_{X}(c) \simeq \frac{\sigma^{2}}{2} c f_{Z}(c)$ which can be estimated for any 
Table 1: Welfare Measures: notation and definition

\begin{tabular}{|l|l|l|}
\hline \multicolumn{1}{|c|}{ Name } & Notation & \multicolumn{1}{c|}{ Definition } \\
\hline $\begin{array}{l}\text { Generalised } \\
\text { entropy index }\end{array}$ & $G E I_{Y}(\alpha)$ & $\frac{1}{\alpha^{2}-\alpha}\left[\frac{\mathcal{L}\left(\infty, y^{\alpha} ; F_{Y}\right)}{\left[\mathcal{L}\left(\infty, y ; F_{Y}\right)\right]^{\alpha}}-1\right], \alpha \notin\{0,1\}$ \\
\hline $\begin{array}{l}\text { Generalised Lorenz } \\
\text { Curve ordinates }\end{array}$ & $\Phi_{Y}(p)$ & $\mathcal{L}\left(Q_{Y}(p), y ; F_{Y}\right)$ \\
\hline $\begin{array}{l}\text { Lorenz } \\
\text { Curve ordinate }\end{array}$ & $\Psi_{Y}(p)$ & {$\left[\mathcal{L}\left(\infty, y ; F_{Y}\right)\right]^{-1} \mathcal{L}\left(Q_{Y}(p), y ; F_{Y}\right)$} \\
\hline $\begin{array}{l}\text { Gini } \\
\text { coefficient }\end{array}$ & $G C_{Y}$ & $1-2 \int_{0}^{1}\left[\mathcal{L}\left(\infty, y ; F_{Y}\right)\right]^{-1} \mathcal{L}\left(Q_{Y}(p), y ; F_{Y}\right) d p$ \\
\hline $\begin{array}{l}\text { FGT class of } \\
\text { poverty indices }\end{array}$ & $P_{Y}(\theta, c)$ & $\mathcal{L}\left(c,\left(\frac{c-y}{c}\right)^{\theta} ; F_{Y}\right) \quad, \theta \geq 0$ \\
\hline
\end{tabular}

potential value of $\sigma^{2}$ plugging in a nonparametric estimate, $\hat{f}_{Z}$, of the density of error contaminated income, evaluated at the chosen poverty line, $c$.

The remainder of the paper is organised as follows. Section 2 develops the approximation to the functional $\mathcal{L}$ and Section 3 applies the approximations to the welfare measures listed in Table 1. Section 4 examines the quality of the approximations comparing them with exact calculations for some specific cases. We find that the approximations are remarkably accurate for a wide range of true income distributions and measurement error distributions even when there is moderate measurement error. Section 5 proposes methods for estimating the functionals appearing in the approximations. A small Monte Carlo experiment suggests that accurate estimation is possible in samples of the size typically found in practical welfare measurement. Section 6 demonstrates the use of the approximations in an illustrative sensitivity analysis of regional inequality measurement using Indonesian household survey data from 1993. Section 7 concludes.

\section{THE APPROXIMATE EFFECT OF MEASUREMENT ERROR}

2.1. Introduction. Calculated welfare measures are statistics which, under suitable conditions (see for example Davidson and Duclos (1997)), are consistent estimators of the same welfare measures computed as functionals of the distribution generating the income survey data. When income data are contaminated by measurement error the distribution from which data are generated differs from the distribution about which we would like to make inferences. So, to understand the way in which measurement error affects views of inequality and poverty gained from welfare measure statistics, the first step is to understand the way in which welfare measure functionals of error free income distributions differ from the same welfare measure functionals applied to error contaminated distributions. For most welfare measures this difference depends upon detailed features of the error free income distribution and the measurement error process 
Table 2: Approximate Error Contaminated Welfare Measures

\begin{tabular}{|l|c|c|c|}
\hline Welfare measure & $\mathcal{W}\left(F_{Z}\right)$ & Approximation: $\mathcal{W}^{A}\left(F_{X}, \sigma^{2}\right)$ & $\mathcal{W}^{A}\left(F_{X}, \sigma^{2}\right) \gtreqless \mathcal{W}\left(F_{X}\right)$ \\
\hline $\begin{array}{l}\text { Generalised } \\
\text { entropy index }\end{array}$ & $G E I_{Z}(\alpha)$ & $G E I_{X}(\alpha)\left(1+\frac{\sigma^{2}}{2} \alpha(\alpha-1)\right)+\frac{\sigma^{2}}{2}$ & $\leq$ \\
\hline $\begin{array}{l}\text { Lorenz Curve } \\
\text { ordinate }\end{array}$ & $\Psi_{Z}(p)$ & $\Psi_{X}(p)-\frac{\sigma^{2}}{2} E(X)^{-1} Q_{X}^{2}(p) f_{X}\left(Q_{X}(p)\right)$ & $\geq$ \\
\hline $\begin{array}{l}\text { Gini } \\
\text { coefficient }\end{array}$ & $G C_{Z}$ & $G C_{X}+\sigma^{2} E(X)^{-1} E\left(X^{2} f_{X}(X)\right)$ & $\geq$ \\
\hline $\begin{array}{l}\text { Headcount } \\
\text { index }\end{array}$ & $P_{Z}(0, c)$ & $P_{X}(0, c)+\frac{\sigma^{2}}{2} c f_{X}(c)\left(\eta_{f_{X}}(c)+2\right)$ & $\geq$ \\
\hline $\begin{array}{l}\text { Poverty } \\
\text { gap }\end{array}$ & $P_{Z}(1, c)$ & $P_{X}(1, c)+\frac{\sigma^{2}}{2} c f_{X}(c)$ & $\geq$ \\
\hline $\begin{array}{l}\text { FGT Index } \\
\text { with } \theta>2\end{array}$ & $P_{Z}(\theta, c)$ & $P_{X}(\theta, c)+\frac{\sigma^{2}}{2} \theta(\theta-1) \times$ & $\geq$ \\
\hline
\end{tabular}

Note: $\eta_{f_{X}}(c)=\left.\nabla_{\log x} \log f_{X}(x)\right|_{x=c}$

and a case by case analysis is required if the impact of measurement error is to be fully understood. Here we derive approximations to the effect of measurement error which only depend on the measurement error variance (in an appropriate metric) and a welfaremeasure-specific functional of error contaminated income.

The methods we employ allow use of a quite general class of measurement error models which includes additive and multiplicative models ${ }^{2}$. However in this paper we consider the simplest form of measurement error that could reasonably be posited for contamination of income data, namely multiplicative measurement error distributed continuously and independently of error free ("true") income. Multiplicative measurement error is a leading case of interest because income is non-negative (at least in the long run). Multiplicative measurement error can also arise when income data are measured relative to an equivalence scale which is itself measured inaccurately ${ }^{3}$. An attractive consequence of the assumption of independence of multiplicative measurement error and true income is the dependence it implies between deviations of error contaminated from error free income $(Z-X)$ and true income $(X)$, larger deviations tending to be associated with larger true income, in the sense that $E[Z-X \mid X]=\lambda X$ for some $\lambda>0$, which is the sort of dependence one would expect to arise in practice.

The relationship between error free income and multiplicative measurement error contaminated income is specified as follows.

$$
\begin{aligned}
Z & =X V \\
V & =e^{-\sigma^{2} / 2} U^{\sigma}
\end{aligned}
$$

\footnotetext{
${ }^{2}$ The GAME model has for, monotonic $\rho(\cdot), \rho(Z)=\rho(X)+\sigma \rho(U)$ and includes simple additive $(\rho(x)=x)$ and multiplicative $(\rho(x)=\log x)$ measurement error models as special cases. Chesher and Schluter (1999) develop the approximations of this paper for the GAME model.

${ }^{3}$ We are grateful to Martin Browning for drawing this to our attention.
} 
Here $\sigma \geq 0$ measures the extent of measurement error with $Z=X$ when $\sigma=0$. The variate $U>0$ has distribution function denoted by $F_{U}(u)$, normalised so that $\log U$ has mean zero and variance one. The scale factor $\exp \left(-\sigma^{2} / 2\right)$ ensures that error contaminated income $(Z)$ and error free income $(X)$ have the same mean to order ${ }^{4} O\left(\sigma^{2}\right)$ as will be shown in Section 2.2. Throughout it is assumed that $X$ and $U$ are continuously distributed.

The distribution function, $F_{Z}$, of error contaminated income is a functional of $F_{X}$ and $F_{U}$ and depends on $\sigma$. For some welfare measures, for example the variance of log income, the error contaminated welfare measure $\mathcal{W}\left(F_{Z}\right)$, and therefore $\mathcal{W}\left(F_{Z}\right)-\mathcal{W}\left(F_{X}\right)$ is determined entirely by $F_{X}$ and $\sigma$. This is unusual, and in most cases the difference in welfare measures for error free and error contaminated income depends upon all aspects of the measurement error distribution $F_{U}$.

To get insight into the impact of measurement error without being specific about the exact distribution of measurement error we consider approximations to error contaminated welfare measures, obtained as second order Taylor series expansions of $\mathcal{W}\left(F_{Z}\right)$ in powers of $\sigma$ around $\sigma=0$. The approximations take the form

$$
\mathcal{W}\left(F_{Z}\right)=\mathcal{W}\left(F_{X}\right)+\sigma^{2} \mathcal{W}^{*}\left(F_{X}\right)+o\left(\sigma^{2}\right)
$$

where $\lim _{\sigma \rightarrow o} O\left(\sigma^{2}\right) / \sigma^{2}=0$ and $\mathcal{W}^{*}(\cdot)$ is a functional determined entirely by $\mathcal{W}(\cdot)$ and independent of the measurement error distribution. There are three points of interest here.

1. The $O\left(\sigma^{2}\right)$ term is determined entirely by $\sigma^{2}$, the error free income distribution and form of the welfare measure. The normalised measurement error distribution $F_{U}$ plays no role except through its variance. Comparison of this term across measures and distributions will show which measures are potentially sensitive to measurement error and for what types of income distribution this sensitivity is high and low.

2. Since $F_{X}$ and $F_{Z}$ differ by $O\left(\sigma^{2}\right)$ it follows that $\sigma^{2} \mathcal{W}^{*}\left(F_{X}\right)$ and $\sigma^{2} \mathcal{W}^{*}\left(F_{Z}\right)$ differ by $o\left(\sigma^{2}\right)$ so that the latter can replace the former in (4) without disturbing the order of the approximation error. This leads to the following, alternative, approximation which has important practical implications.

$$
\mathcal{W}\left(F_{Z}\right)=\mathcal{W}\left(F_{X}\right)+\sigma^{2} \mathcal{W}^{*}\left(F_{Z}\right)+o\left(\sigma^{2}\right)
$$

3. Error contaminated data, that is, realisations of $Z$, allow estimation of $F_{Z}$, and so, of $\mathcal{W}^{*}\left(F_{Z}\right)$. This leads to new tools for improved welfare measurement and comparison in real world policy analysis in which measurement error is a non-ignorable, everpresent feature.

(a) With an estimate $\mathcal{W}^{*}\left(\hat{F}_{Z}\right)$, the sensitivity of welfare measurements to alternative amounts $\left(\sigma^{2}\right)$ of measurement error can be assessed. One may find that, for the chosen welfare measure and error contaminated distribution, $\mathcal{W}^{*}\left(\hat{F}_{Z}\right)$ is small enough to be ignored for plausible amounts of measurement error.

\footnotetext{
${ }^{4}$ In the sense that $E[Z]-E[X]=o\left(\sigma^{2}\right)$ where $\lim _{\sigma \rightarrow 0} o\left(\sigma^{2}\right) / \sigma^{2}=0$.
} 
(b) It is possible to calculate an approximately corrected welfare measure using $\widehat{\mathcal{W}\left(F_{X}\right)}=\mathcal{W}\left(\hat{F}_{Z}\right)-\hat{\sigma}^{2} \mathcal{W}^{*}\left(\hat{F}_{Z}\right)$, where $\hat{\sigma}^{2}$ is an extraneous estimate, $\hat{\sigma}^{2}$, or for $\hat{\sigma}^{2}$ passing through a range of plausible values.

(c) Estimates, $\widehat{\mathcal{W}\left(F_{X}\right)}$, under different amounts of measurement error can be compared across groups over which there might be expected to be different amounts of measurement error, for example rural and urban households. One would be concerned for the appropriateness of policy targeting if rankings of groups were sensitive to plausible variations across groups in the amount of measurement error.

We now develop the tools required to obtain the approximations to the welfare measure functionals, first considering the relationship between error free and error contaminated income distributions.

\subsection{Approximate density functions under multiplicative measurement error.}

Chesher (1991) shows that in the additive measurement error model

$$
R=S+\sigma V
$$

where $V$ is continuously distributed independently of $S$ with mean zero and variance one, the density of $R$ is

$$
f_{R}(r) \simeq f_{S}(r)+\frac{\sigma^{2}}{2} f_{S}^{\prime \prime}(r)
$$

where " $\simeq$ " indicates a difference between left and right hand sides that is $o\left(\sigma^{2}\right)$ where $\lim _{\sigma \rightarrow 0} o\left(\sigma^{2}\right) / \sigma^{2}=0$.

The result follows directly from a second order Taylor series expansion of the marginal density of error contaminated $R$,

$$
f_{R}(r)=\int_{-\infty}^{\infty} f_{S}(r-\sigma v) f_{V}(v) d v
$$

in powers of $\sigma$ around $\sigma=0$. The approximation holds with remainder term of the order indicated if (a) $f_{S}(s)$ has bounded third derivative and (b) $V$ has finite third absolute moment around zero. The approximation error expressed here and later as $o\left(\sigma^{2}\right)$ is in fact of order $O\left(\sigma^{3}\right)$ or smaller and is $O\left(\sigma^{4}\right)$ if $V$ is symmetrically distributed with bounded fourth moment.

Let

$$
W=X U^{\sigma}
$$

and define $R, S$ and $V$ in (6) as logarithms of respectively $W, X$ and $U$. Then using $f_{W}(w)=w^{-1} f_{R}(\log w)$ and $(7)$ gives

$$
f_{W}(w) \simeq w^{-1}\left(f_{S}(\log w)+\frac{\sigma^{2}}{2} f_{S}^{\prime \prime}(\log w)\right)
$$


and expressing the right hand side of this approximation in terms of the density of $X$, and its derivatives yields

$$
f_{W}(w) \simeq f_{X}(w)+\frac{\sigma^{2}}{2}\left(w^{2} f_{X}^{\prime \prime}(w)+3 w f_{X}^{\prime}(w)+f_{X}(w)\right) .
$$

Finally, defining $Z=\exp \left(-\sigma^{2} / 2\right) W$, which introduces the mean correction so that $E[Z] \simeq$ $E[X]$, noting that

$$
\begin{aligned}
f_{Z}(z) & =e^{\sigma^{2} / 2} f_{W}\left(e^{\sigma^{2} / 2} z\right) \\
& \simeq f_{W}(z)+\frac{\sigma^{2}}{2}\left(f_{W}(z)+z f_{W}^{\prime}(z)\right),
\end{aligned}
$$

substituting in (8) and retaining terms of order $O\left(\sigma^{2}\right)$ gives the approximate error contaminated density, as follows.

$$
\begin{aligned}
f_{Z}(z) \simeq f_{Z}^{a}(z) & =f_{X}(z)+\frac{\sigma^{2}}{2}\left(z^{2} f_{X}^{\prime \prime}(z)+4 z f_{X}^{\prime}(z)+2 f_{X}(z)\right) \\
& =f_{X}(z)+\frac{\sigma^{2}}{2} \frac{d^{2}}{d z^{2}}\left(z^{2} f_{X}(z)\right)
\end{aligned}
$$

Details are given in Chesher, Dumangane and Smith (2001).

2.3. Approximate welfare measure functionals. For all the welfare measures considered in this paper (see Table 1 ) the approximate effect of measurement error on welfare measure functionals is determined by its effect on the functional $\mathcal{L}(a, N ; F)$ given in equation (1). This effect is now derived. Applying the functional to the error contaminated income distribution gives

$$
\mathcal{L}\left(a_{Z}, N ; F_{Z}\right)=\int_{0}^{a_{Z}} N\left(z, a_{Z}\right) d F_{Z}(z) .
$$

The limit of integration may be distribution dependent and to allow for this write the limit of integration as $a_{Z}=\mathcal{A}\left(F_{Z}\right)$, let $a_{X}=\mathcal{A}\left(F_{X}\right)$, and assume $a_{Z}$ admits a first order Taylor series expansion in $\sigma^{2}$ as follows: $a_{Z} \simeq a_{X}+\sigma^{2} \mathcal{A}_{1}\left(F_{X}\right)$. Substituting this approximation for $a_{Z}$ and the density approximation, equation (9), gives the following.

$$
\mathcal{L}\left(a_{Z}, N ; F_{Z}\right) \simeq\left\{\int_{0}^{a_{X}}+\int_{a_{X}}^{a_{X}+\sigma^{2} \mathcal{A}_{1}\left(F_{X}\right)}\right\} N\left(z, a_{Z}\right) f_{Z}^{a}(z) d z
$$

Expanding $N\left(z, a_{Z}\right)$ and neglecting terms of order $o\left(\sigma^{2}\right)$ the following approximation is obtained.

$$
\begin{aligned}
\mathcal{L}\left(a_{Z}, N ; F_{Z}\right) \simeq & \mathcal{L}\left(a_{X}, N ; F_{X}\right)+\sigma^{2} \mathcal{A}_{1}\left(F_{X}\right) N\left(a_{X}, a_{X}\right) f_{X}\left(a_{X}\right) \\
& +\sigma^{2} \mathcal{A}_{1}\left(F_{X}\right) \int_{0}^{a_{X}} N_{01}\left(x, a_{X}\right) f_{X}(x) d x \\
& +\frac{\sigma^{2}}{2} \int_{0}^{a_{X}} N\left(x, a_{X}\right) \frac{d^{2}}{d x^{2}}\left(x^{2} f_{X}(x)\right) d x
\end{aligned}
$$


Here and later $N_{i j}(y, a)$ denotes the $i+j$ order partial derivative $\nabla_{y}^{i} \nabla_{a}^{j} N(y, a)$. These derivatives must be bounded for all $i$ and $j$ with $i+j=2$ for this approximation to hold.

The leading term in equation (10) is the functional applied to the error free distribution. The remaining terms capture the first order effect of measurement error. Of these, the first term arises because of the potential distribution dependence of the limit of integration, the second term arises because of the potential appearance of this distribution dependent limit in the function $N$ and the final term arises directly from error contamination of the density of $X$.

Under suitable restrictions on the behaviour of $N\left(x, a_{X}\right)$ and $f_{X}(x)$ as $x \rightarrow 0$, the last term can be integrated by parts to give the following approximation ${ }^{5}$.

$$
\begin{aligned}
\mathcal{L}\left(a_{Z}, N ; F_{Z}\right) \simeq & \mathcal{L}\left(a_{X}, N ; F_{X}\right)+\sigma^{2} \mathcal{A}_{1}\left(F_{X}\right) N\left(a_{X}, a_{X}\right) f_{X}\left(a_{X}\right) \\
& +\sigma^{2} \mathcal{A}_{1}\left(F_{X}\right) \int_{0}^{a_{X}} N_{01}\left(x, a_{X}\right) f_{X}(x) d x \\
& +\frac{\sigma^{2}}{2}\left\{N\left(a_{X}, a_{X}\right) a_{X}^{2} f_{X}^{\prime}\left(a_{X}\right)+\left[2 N\left(a_{X}, a_{X}\right) a_{X}-N_{10}\left(a_{X}, a_{X}\right) a_{X}^{2}\right] f_{X}\left(a_{X}\right)\right\} \\
& +\frac{\sigma^{2}}{2} \int_{0}^{a_{X}} N_{11}\left(x, a_{X}\right) x^{2} f_{X}(x) d x .
\end{aligned}
$$

This approximation is employed in the remainder of the paper.

\section{APPROXIMATIONS FOR PARTICULAR WELFARE MEASURES}

3.1. Introduction. In this Section explicit forms of the approximations (10) and (11) are provided for some commonly used welfare measures. Moments and quantiles, which figure in a number of the welfare measures, are considered first, and then, in Sections 3.3 and 3.4, the approximate effects of measurement error on the Generalised Entropy Index, the Lorenz curve and the Gini coefficient are examined. Section 3.5 considers poverty indices, first with distribution independent poverty lines, and then with distribution dependent poverty lines.

3.2. Moments and quantiles. Moments, functions of moments, and quantiles appear in a number of the welfare measures and it is useful to have approximations of these to hand. Using equation (11) with $a=\infty, N(y)=y^{\alpha}$ and suitable assumptions on the tail behaviour of $f_{X}$ yields

$$
E\left[Z^{\alpha}\right] \simeq E\left[X^{\alpha}\right]\left(1+\frac{\sigma^{2}}{2} \alpha(\alpha-1)\right)
$$

which implies, for the mean and its inverse,

$$
E[Z] \simeq E[X] \quad E[Z]^{-1} \simeq E[X]^{-1} .
$$

\footnotetext{
${ }^{5}$ The required conditions are $\lim _{x \rightarrow 0} x N\left(x, a_{X}\right) f_{X}(x)=0, \lim _{x \rightarrow 0} x^{2} N\left(x, a_{X}\right) f_{X}^{\prime}(x)=0$, $\lim _{x \rightarrow 0} x^{2} N_{10}\left(x, a_{X}\right) f_{X}(x)=0$.
} 
The quantile function is defined implicitly by $p=F_{Z}\left(Q_{Z}(p)\right)=F_{X}\left(Q_{X}(p)\right)$. Consider equation (11) with $N(y)=1$, let $a_{Z}=Q_{Z}(p)$ which has Taylor series approximation

$$
Q_{Z}(p) \simeq Q_{X}(p)+\sigma^{2} \mathcal{A}_{1}\left(F_{X}\right)
$$

where

$$
\mathcal{A}_{1}\left(F_{X}\right)=\left.\nabla_{\sigma^{2}} Q_{Z}(p)\right|_{\sigma^{2}=0}
$$

which is now derived. Since $\mathcal{L}\left(Q_{Z}(p), 1 ; F_{Z}\right)=\mathcal{L}\left(Q_{X}(p), 1 ; F_{X}\right)=p$, upon substituting in (11) we have

$$
p \simeq p+\sigma^{2} f_{X}\left(Q_{X}(p)\right) \mathcal{A}_{1}\left(F_{X}\right)+\frac{\sigma^{2}}{2}\left\{Q_{X}^{2}(p) f_{X}^{\prime}\left(Q_{X}(p)\right)+2 Q_{X}(p) f_{X}\left(Q_{X}(p)\right)\right\}
$$

giving

$$
\begin{aligned}
\mathcal{A}_{1}\left(F_{X}\right) & =-\frac{1}{2} \frac{Q_{X}^{2}(p) f_{X}^{\prime}\left(Q_{X}(p)\right)+2 Q_{X}(p) f_{X}\left(Q_{X}(p)\right)}{f_{X}\left(Q_{X}(p)\right)} \\
& =-\frac{1}{2} Q_{X}(p)\left(\eta_{f_{X}}\left(Q_{X}(p)\right)+2\right)
\end{aligned}
$$

where

$$
\eta_{f_{X}}(a)=\left.\nabla_{\log x} \log f_{X}(x)\right|_{x=a}
$$

is the elasticity of the income density at $x=a$. Substituting in (14) the following approximation to the quantile function under measurement error contamination is obtained.

$$
Q_{Z}(p) \simeq Q_{X}(p)\left[1-\frac{\sigma^{2}}{2}\left(\eta_{f_{X}}\left(Q_{X}(p)\right)+2\right)\right]
$$

To the order of approximation considered, error contaminated quantiles exceed error free quantiles over all intervals in which the elasticity of the density function of true income is less than -2 . Clearly these intervals must be in regions where the density function is falling. At all $p$ values for which the density is increasing error contaminated quantiles lie below error free quantiles.

The distortion of the quantile function produced by measurement error has consequences for the "first order" approach to comparisons of distributions. Consider the class of social welfare functions $\int u(y) d F(y)$ where the utility function $u$ is increasing, and two distribution functions $F_{S}$ and $F_{T}$. A well-known dominance result (Saposnik (1981, 1983)) states that $Q_{S}(p) \geq Q_{T}(p)$ for all $p$ if and only if social welfare associated with distribution $F_{S}$ exceeds that associated with $F_{T}$ for all utility functions $u$ in the admissible class. Equation (15) shows that error free and error contaminated income distribution functions typically cross. So, when income distributions subject to differing amounts of measurement error are compared without consideration of the impact of measurement error, it is possible to conclude that there is no first order dominance when in fact there is dominance for error free income. Estimation of components of a measurement error correction, as illustrated in Section 5, allows the potential impact of measurement error on first order comparisons to be assessed. 
3.3. The Generalised Entropy Index. The Generalised Entropy Index (GEI) is of interest because any inequality index which satisfies the principle of transfer, scale invariance and decomposability must be ordinally equivalent to the GEI (Cowell $(1980))^{6}$.

The moment approximations given earlier imply

$$
E\left[Z^{\alpha}\right] E[Z]^{-\alpha} \simeq E\left[X^{\alpha}\right] E[X]^{-\alpha}\left(1+\frac{\sigma^{2}}{2} \alpha(\alpha-1)\right)
$$

which implies

$$
G E I_{Z}(\alpha) \simeq G E I_{X}(\alpha)\left(1+\frac{\sigma^{2}}{2} \alpha(\alpha-1)\right)+\frac{\sigma^{2}}{2} .
$$

To the order of approximation considered, the GEI is larger for error contaminated income distributions than for error free distributions whenever $\alpha(\alpha-1)>-G E I_{X}(\alpha)^{-1}$, which holds for all $G E I_{X}(\alpha)$ when $\alpha<0$ and $\alpha>1$, and for all $\alpha$ when $G E I_{X}(\alpha)<4$. Note that 4 is an unusually large value for this index in practice for $\alpha \in(0,1)$. The first order effect of measurement error on the GE Index is invariant with respect to the form of the error free income distribution.

3.4. The Lorenz curve and the Gini coefficient. The "second order" approach to distributional comparison considers the class of social welfare functions $\int u(y) d F(y)$ where the utility function $u$ is increasing and concave, and two distribution functions $F_{S}$ and $F_{T}$. Atkinson (1970) shows that, when $S$ and $T$ have the same mean, all measures satisfying symmetry, mean independence and the principle of transfers will register a greater inequality for $F_{T}$ than for $F_{S}$ if and only if the Lorenz curve associated with $F_{S}$ lies everywhere above that associated with $F_{T}$. The theorem has been extended by Shorrocks (1983) to distributions with different means: social welfare associated with distribution $F_{S}$ is greater than that associated with $F_{T}$ if and only if the Generalised Lorenz curve for $F_{S}$ lies everywhere above that of $F_{T}$. It is therefore of interest to study the impact of measurement error on Lorenz curves.

To derive the effect of measurement error on the Lorenz curve ordinate, first consider the Generalised Lorenz curve ordinate $\mathcal{L}\left(Q_{Z}(p), z ; F_{Z}\right)$. Exploiting the quantile approximation (15) which gives the form of the term $\mathcal{A}_{1}\left(F_{X}\right)$ that appears in (11) we have the following.

$$
\Phi_{Z}(p)=\mathcal{L}\left(Q_{Z}(p), z ; F_{Z}\right) \simeq \Phi_{X}(p)-\frac{\sigma^{2}}{2} Q_{X}^{2}(p) f_{X}\left(Q_{X}(p)\right)
$$

Since $E[Z]^{-1} \simeq E[X]^{-1}$, the simple Lorenz curve ordinate is

$$
\Psi_{Z}(p) \simeq \Psi_{X}(p)-\frac{\sigma^{2}}{2} E(X)^{-1} Q_{X}^{2}(p) f_{X}\left(Q_{X}(p)\right)
$$

\footnotetext{
${ }^{6}$ One such ordinally equivalent index is the Atkinson index (Atkinson (1970)) which is therefore not considered specifically here.
} 
The first order effect of measurement error is to push the Lorenz curve outwards, so the Gini coefficient must be increased.

The Gini coefficient for the distribution of error contaminated income is

$$
G C_{Z}=1-2 \int_{0}^{1} \Psi_{Z}(p) d p
$$

Using the approximation (17) and integrating with respect to $Q_{X}(p)$ in place of $p$ leads directly to

$$
G C_{Z} \simeq G C_{X}+\sigma^{2} \frac{E\left[X^{2} f_{X}(X)\right]}{E[X]}
$$

To the order of approximation considered, the Gini coefficient for the distribution of error contaminated income is larger than the Gini coefficient for the distribution of error free income. The increase caused by measurement error is smaller when the density of $X$ is high-valued close to zero and low-valued far from zero, for example when the income distribution is heavily positively skewed.

3.5. Poverty indices. A large class of poverty indices (see Foster, Greer and Thorbecke (1984)) have the form

$$
P_{Z}(\theta, c)=\int_{0}^{c}\left(1-\frac{y}{c}\right)^{\theta} d F_{Y}(y)=\mathcal{L}\left(c,\left(1-\frac{y}{c}\right)^{\theta} ; F_{Y}\right)
$$

where $\theta \geq 0$ is a sensitivity parameter and $c$ is the poverty line. In some applications the poverty line is distribution dependent, for example specified as a fraction of median income. First consider the case in which the poverty line is distribution independent.

Distribution independent poverty lines. Care is required in producing approximations to these poverty indices because the integration by parts done to reach (11) is not valid when $\theta<2$. However the approximation (10) is valid for all $\theta>0$, giving the following approximation.

$$
P_{Z}(\theta, c)=P_{X}(\theta, c)\left(1+\sigma^{2}\right)+\frac{\sigma^{2}}{2} \int_{0}^{c}\left(1-\frac{x}{c}\right)^{\theta}\left(x^{2} f_{X}^{\prime \prime}(x)+4 x f_{X}^{\prime}(x)\right) d x .
$$

When $\theta=0$ we have the head count index $\mathcal{L}\left(c, 1, F_{Z}\right)$ and direct application of $(10)$ with $N\left(z, a_{Z}\right)=1$, gives

$$
\begin{aligned}
P_{Z}(0, c) & \simeq P_{X}(0, c)+\frac{\sigma^{2}}{2}\left(c^{2} f_{X}^{\prime}(c)+2 c f_{X}(c)\right) \\
& =P_{X}(0, c)+\frac{\sigma^{2}}{2} c f_{X}(c)\left(\eta_{f_{X}}(c)+2\right)
\end{aligned}
$$

where in the second line $\eta_{f_{X}}(c)$ is the elasticity of the income density at the poverty line. This mirrors the quantile approximation given in Section 3.2. To the order considered here the head count index for the error contaminated distribution exceeds that for the 
error free distribution whenever $\eta_{f_{X}}(c)>-2$. So, if the poverty line is drawn where the income density is rising, for example to the left of a mode of the income density, then measurement error causes the head count index to rise. However for a poverty line drawn where the income density is falling it may be possible for measurement error to cause the head count index to fall. This could perhaps occur in cases in which the mode of the income density is far to the left of the main mass of the distribution.

When $\theta=1$, one round of integration by parts can be done and this is sufficient to give the following simple approximation.

$$
P_{Z}(1, c) \simeq P_{X}(1, c)+\frac{\sigma^{2}}{2} c f_{X}(c)
$$

In this case, to the order considered the error contaminated index is larger than the error free index for all income distributions and choices of distribution free poverty line. This is also the case for all $\theta \geq 2$ when (11) is valid, and since $N(c, c)=N_{10}(c, c)=0$, we obtain

$$
P_{Z}(\theta, c) \simeq P_{X}(\theta, c)+\frac{\sigma^{2}}{2} \theta(\theta-1) \int_{0}^{c}\left(\frac{z}{c}\right)^{2}\left(1-\frac{z}{c}\right)^{\theta-2} f_{X}(z) d z
$$

which, after some manipulation can be written as

$$
P_{Z}(\theta, c) \simeq P_{X}(\theta, c)+\frac{\sigma^{2}}{2} \theta(\theta-1)\left[P_{X}(\theta, c)-2 P_{X}(\theta-1, c)+P_{X}(\theta-2, c)\right]
$$

The integral in (20) is positive, so for $\theta \geq 2$ the error contaminated index is always larger than the error free index ${ }^{7}$.

Distribution dependent poverty lines. Now let the poverty line be distribution dependent. There are many possible specifications but one commonly encountered specifies the poverty line as a function of a $p$-quantile (for example a fraction of the median), $c_{Z}=c\left(Q_{Z}(p)\right)$, with $c_{X}=c\left(Q_{X}(p)\right)$.

In the case of the head count index, since $N\left(x, a_{X}\right)=1$, the term in (11) involving $N_{01}\left(x, a_{X}\right)$ is absent and the following approximation is obtained.

$$
P_{Z}\left(0, c_{Z}\right) \simeq P_{X}\left(0, c_{X}\right)+\frac{\sigma^{2}}{2} c_{X} f_{X}\left(c_{X}\right)\left(\eta_{f_{X}}\left(c_{X}\right)+2-\eta_{c}\left(Q_{X}(p)\right)\left(\eta_{f_{X}}\left(Q_{X}(p)\right)+2\right)\right)
$$

Here $\eta_{c}$ is the elasticity of the poverty line $c\left(Q_{X}(p)\right)$ with respect to $Q_{X}(p)$. In all practical applications $c(\cdot)$ will be increasing, in which case the additional term arising because the poverty line is distribution dependent will tend to reduce the impact of measurement error as long as $c_{X}=c\left(Q_{X}(p)\right)$ and $Q_{X}(p)$ are close enough that $\eta_{f_{X}}\left(c_{X}\right)+2$ and $\eta_{f_{X}}\left(Q_{X}(p)\right)+2$ have the same $\operatorname{sign}^{8}$.

\footnotetext{
${ }^{7}$ For $\theta$ in $[0,1) \cup(1,2)$ it appears that the sign of the approximation to $P_{Z}(\theta, c)-P_{X}(\theta, c)$ depends upon the location of the poverty line, $c$, and on the error free income density as is the case for $\theta=0$ (see equation (18)). It does not seem possible to $\operatorname{sign} P_{Z}(\theta, c)-P_{X}(\theta, c)$ for $\theta<2$ except when $\theta=1$.

${ }^{8}$ Of course if $c_{Z}=c\left(Q_{Z}(p)\right)$ and $Q_{Z}(p)$ are coincident then the $O\left(\sigma^{2}\right)$ disappears altogether because in this trivial case $P_{Z}\left(0, c_{Z}\right)=P_{Z}\left(0, Q_{Z}(p)\right)=p$ exactly.
} 
Now consider poverty indices with $\theta>0$ and a distribution dependent poverty line. Here

$$
N_{01}\left(x, c_{X}\right)=\theta \frac{x}{c_{X}^{2}}\left(1-\frac{x}{c_{X}}\right)^{\theta-1}
$$

and, for $\theta \geq 1$, after some manipulation we obtain the term, $\Lambda$, to be added to the approximations to allow for distribution dependence in the poverty line.

$$
\Lambda\left(Q_{X}(p), c(\cdot)\right) \simeq \frac{\sigma^{2}}{2} \theta\left(\eta_{f_{X}}\left(Q_{X}(p)\right)+2\right) \eta_{c}\left(Q_{X}(p)\right)\left(P_{X}\left(\theta, c_{X}\right)-P_{X}\left(\theta-1, c_{X}\right)\right)
$$

Since $P_{X}\left(\theta, c_{X}\right)$ is a decreasing function of $\theta$ and in practice $c(\cdot)$ will be an increasing function, $\Lambda$ will be positive when the density elasticity at the poverty line, $\eta_{f_{X}}\left(Q_{X}(p)\right)$, exceeds -2 . For $\theta \in(0,1)$ the integral in (11) involving $N_{01}\left(x, a_{X}\right)$ does not converge and the small variance approximation used here is in this case not available.

\section{The ACCURACY of the APproximations}

We now examine the accuracy of the approximations. We do this by computing the exact distributions of error contaminated income for a variety of combinations of error free income and measurement error distributions and differing amounts of measurement error. We then calculate welfare measures using these exact distributions and compare them with the approximations ${ }^{9}$. Since we compare the error contaminated welfare measures with what is obtained when there is no measurement error, this exercise also gives a feeling for the potential magnitude of the distortions to welfare measures caused by measurement error.

For this purpose we work in a flexible framework and consider a variety of cases in which log error free income and log measurement error are independently distributed with exponential power (EP) distributions (see Box and Tiao (1973)). A random variable $V \in(-\infty, \infty)$ with an EP distribution with mean $\mu$, scale parameter $\tau>0$ and shape parameter $\beta \in(-1,1]$ has density function proportional to $\exp \left\{-\tau^{-1}|v-\mu|^{2 /(1+\beta)}\right\}$. Nine combinations of measurement error distribution and error free income distribution are considered, namely combinations of:

- three EP distributions for zero mean log measurement error with $\beta_{M E}=-0.9$ (close to uniform), $\beta_{M E}=0.0$ (Gaussian) and $\beta_{M E}=+1.0$ (Laplace), and,

- three EP distributions for log error free income with $\beta_{I N C} \in\{-0.5,0.0,+0.5\}$.

Poverty indexes are studied first, then Lorenz curve ordinates and the Gini coefficient.

\footnotetext{
${ }^{9}$ Exact error contaminated income distributions and the required functionals of them were computed numerically using the Gauss-Kronrod quadrature method as implemented in the NIntegrate function of Mathematica 4.0 (Wolfram (1999).
} 
4.1. Poverty Indexes. We consider poverty lines, $c$, drawn so that for each of the three error free income distributions the headcount index is 0.20 . Figure 1,2 and 3 refer to respectively the head count index, $P_{Y}(0, c)$, the poverty gap index, $P_{Y}(1, c)$ and the poverty severity index, $P_{Y}(2, c)$. In each figure there are nine panes showing the ratio of the exact (dashed) and approximate ${ }^{10}$ (solid) error contaminated indexes to the value of the error free index as measurement error varies so that the excess log variance ratio, $\nu=\operatorname{Var}[\log Z] / \operatorname{Var}[\log X]$, varies from 1 to 1.25 . Each pane relates to a distinct combination of error free income distribution and measurement error distribution.

Looking first at the dashed lines in these graphs, it can be seen that for all distributional combinations and choice of index the impact of measurement error on the index is substantial, varying close to linearly with the excess log variance ratio. An excess log variance ratio of 1.25 causes the headcount, poverty gap and poverty severity indexes to be inflated by around $25 \%, 50 \%$ and $80 \%$ respectively relative to their values for error free income. Looking at the solid lines on the graphs it can be seen that the approximations to these significant measurement error effects are very good indeed. In no case do the approximations deviate by more than $4 \%$ from the exact values. In most cases the deviations are far smaller than this.

Figure 4 compares bias corrected indexes with values of the indexes for error free distributions, plotting $\left(\mathcal{W}\left(F_{Z}\right)-\sigma^{2} \mathcal{W}^{*}\left(F_{Z}\right)\right) / \mathcal{W}\left(F_{X}\right)$ as a function of the excess log variance ratio. In all cases this ratio lies close to 1 indicating that the approximations deliver accurate measurement error corrections under a wide variety of conditions.

4.2. Lorenz curves and Gini coefficients. Figure 5 shows the difference between error free Lorenz curve ordinates and ordinates of exact and approximate error contaminated Lorenz curves when the excess log variance ratio is 1.2. For proportions of income away from 0 and 1 the difference is of course always positive, in the cases considered here peaking at between 0.03 and 0.05 for income proportions around 0.8 . Variations in the shape parameter of the measurement error distribution (compare columns in Figure 5) have little effect compared with variations in the shape parameter of the error free income distribution (compare rows). In all cases the approximations deliver values that are very close indeed to the exact Lorenz curve ordinates ${ }^{11}$.

It comes as no surprise then to find that the approximations to the Gini coefficients are also accurate. Figure 6 shows exact and approximate error contaminated Gini coefficients expressed as ratios to values of the Gini Coefficient for error free data for excess lag variance ratios varying from 1.0 to 1.2. Measurement error on this scale causes inflation of the Gini coefficient by up to 8\% (see the dashed lines in Figure 6), a magnitude very accurately picked up by the approximations.

\footnotetext{
${ }^{10}$ The form of the approximation used here employs $F_{Z}$ rather than $F_{X}$ in $\mathcal{W}^{*}(\cdot)$ since this is the form more likely to be employed in applications, as is done in Section 6 .

${ }^{11}$ The approximations vary very slightly as the measurement error distribution shape parameter is changed because they are calculated using $\mathcal{W}^{*}\left(F_{Z}\right)$ and $F_{Z}$ alters as the measurement error distribution changes.
} 
Figure 1: Poverty index $P(0, c)$ with poverty line, $c$, at the 20th percentile of the error free income distribution. Approximate (solid) and exact (dashed) error contaminated index relative to error free index. Shape parameters: error free income $\beta_{I N C}$, measurement error $\beta_{M E}$.
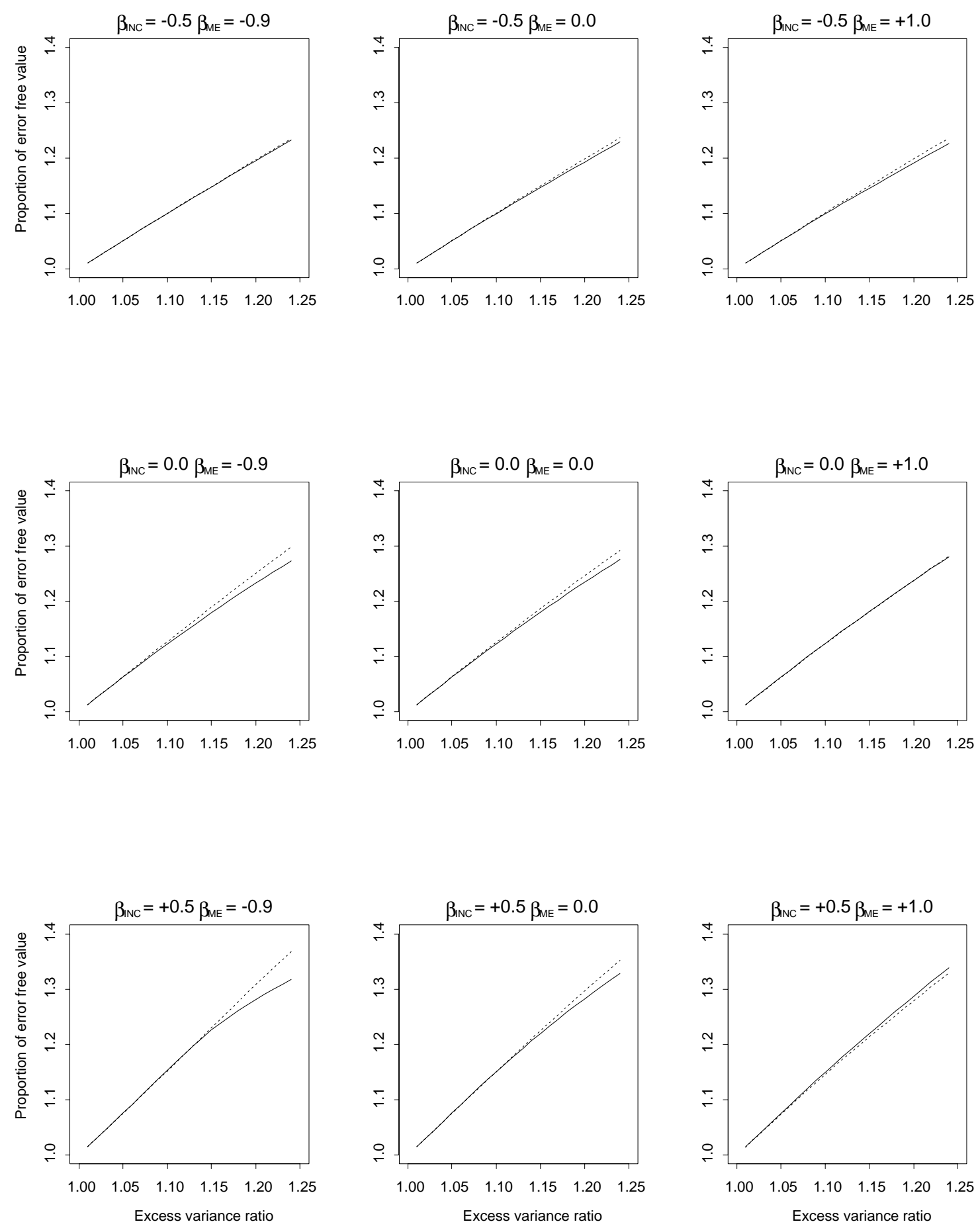
Figure 2: Poverty index $P(1, c)$ with poverty line, $c$, at the 20th percentile of the error free income distribution. Approximate (solid) and exact (dashed) error contaminated index relative to error free index. Shape parameters: error free income $\beta_{I N C}$, measurement error $\beta_{M E}$.
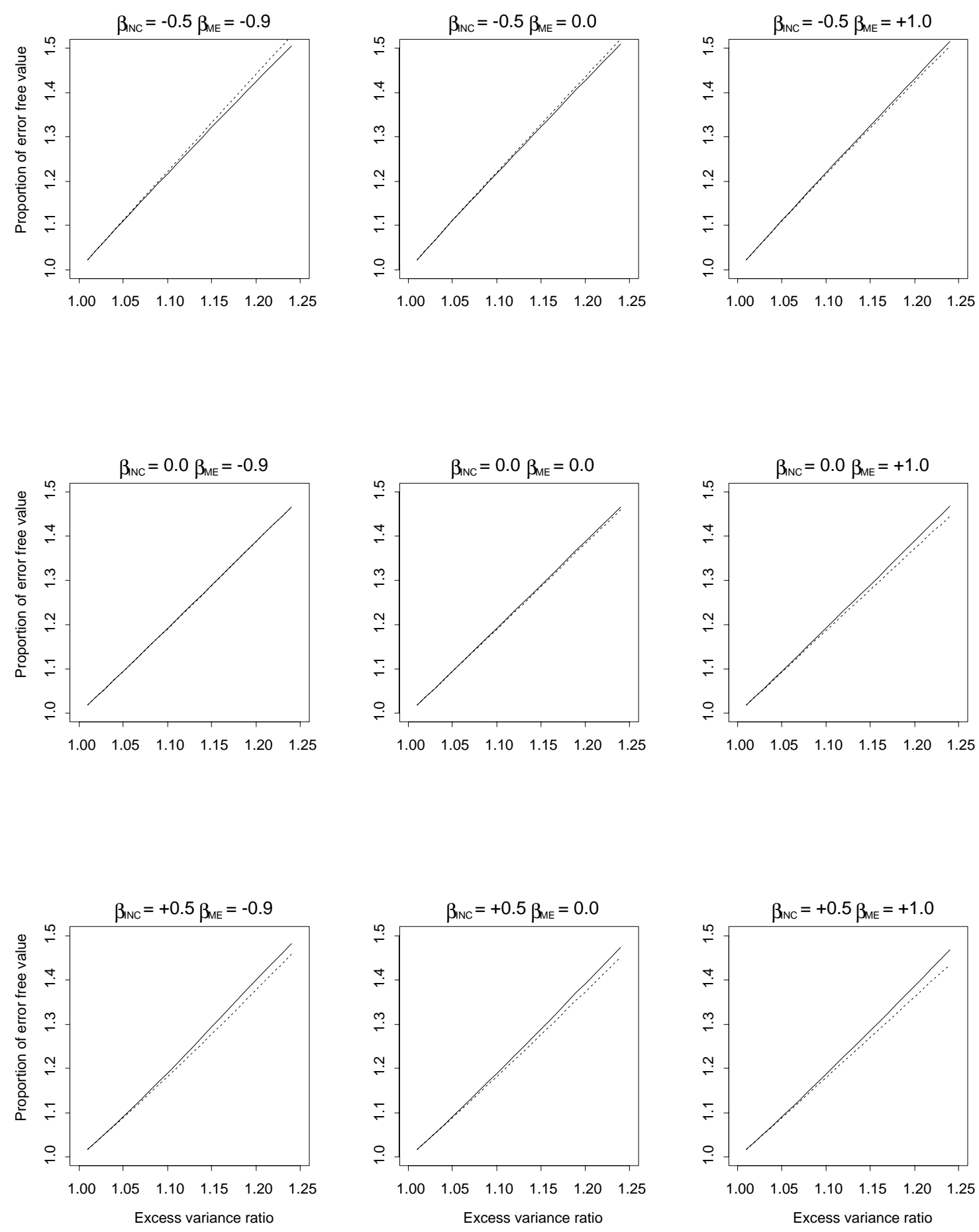
Figure 3: Poverty index $P(2, c)$ with poverty line, $c$, at the 20th percentile of the error free income distribution. Approximate (solid) and exact (dashed) error contaminated index relative to error free index. Shape parameters: error free income $\beta_{I N C}$, measurement error $\beta_{M E}$.
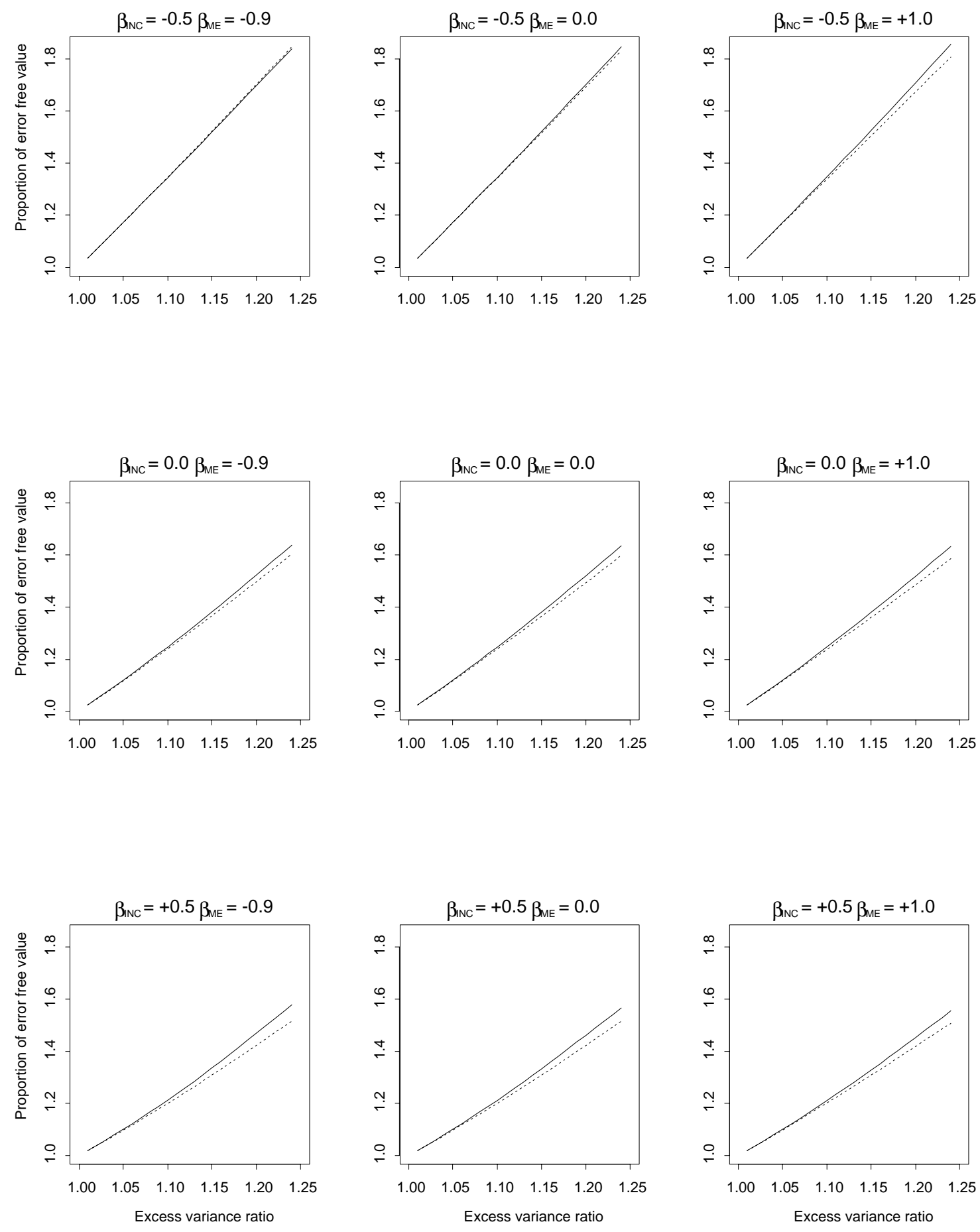
Figure 4: Ratios of corrected measurement error contaminated poverty indexes to exact error free indexes for measurement error distributions with shape parameters: $\beta_{M E}=$ -0.9 (near uniform, solid), $\beta_{M E}=0.0$ (Gaussian, dotted), $\beta_{M E}=+1.0$ (Laplace, dashed). Poverty line drawn at the 20th perecntile of error free income.
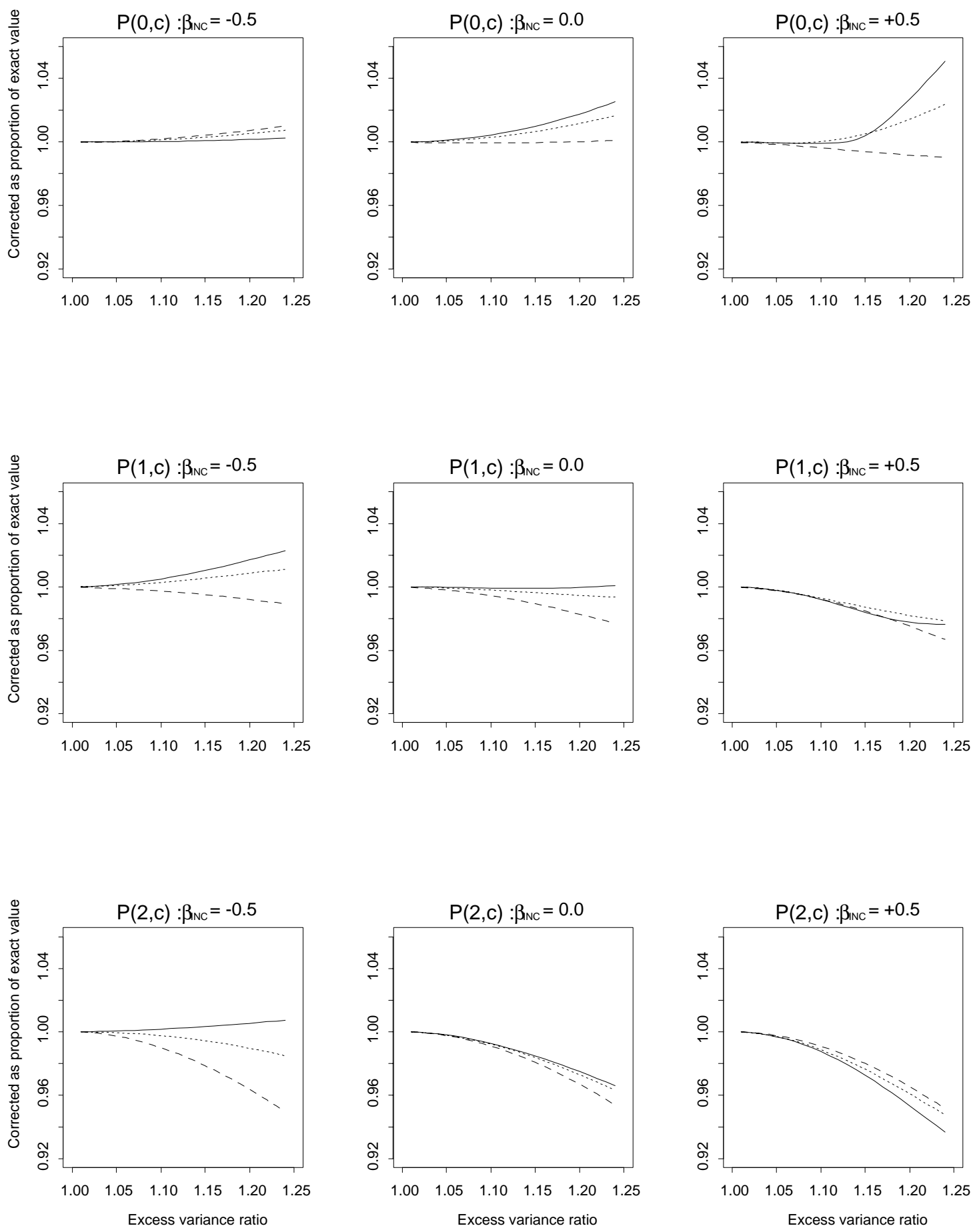
Figure 5: Difference between Lorenz curve ordinates when the excess log variance ratio is 1.2 , ordinates with no measurement error minus approximate (solid) and exact (dashed) ordinates of error contaminated Lorenz curves. Shape parameters: error free income $\beta_{I N C}$, measurement error $\beta_{M E}$.
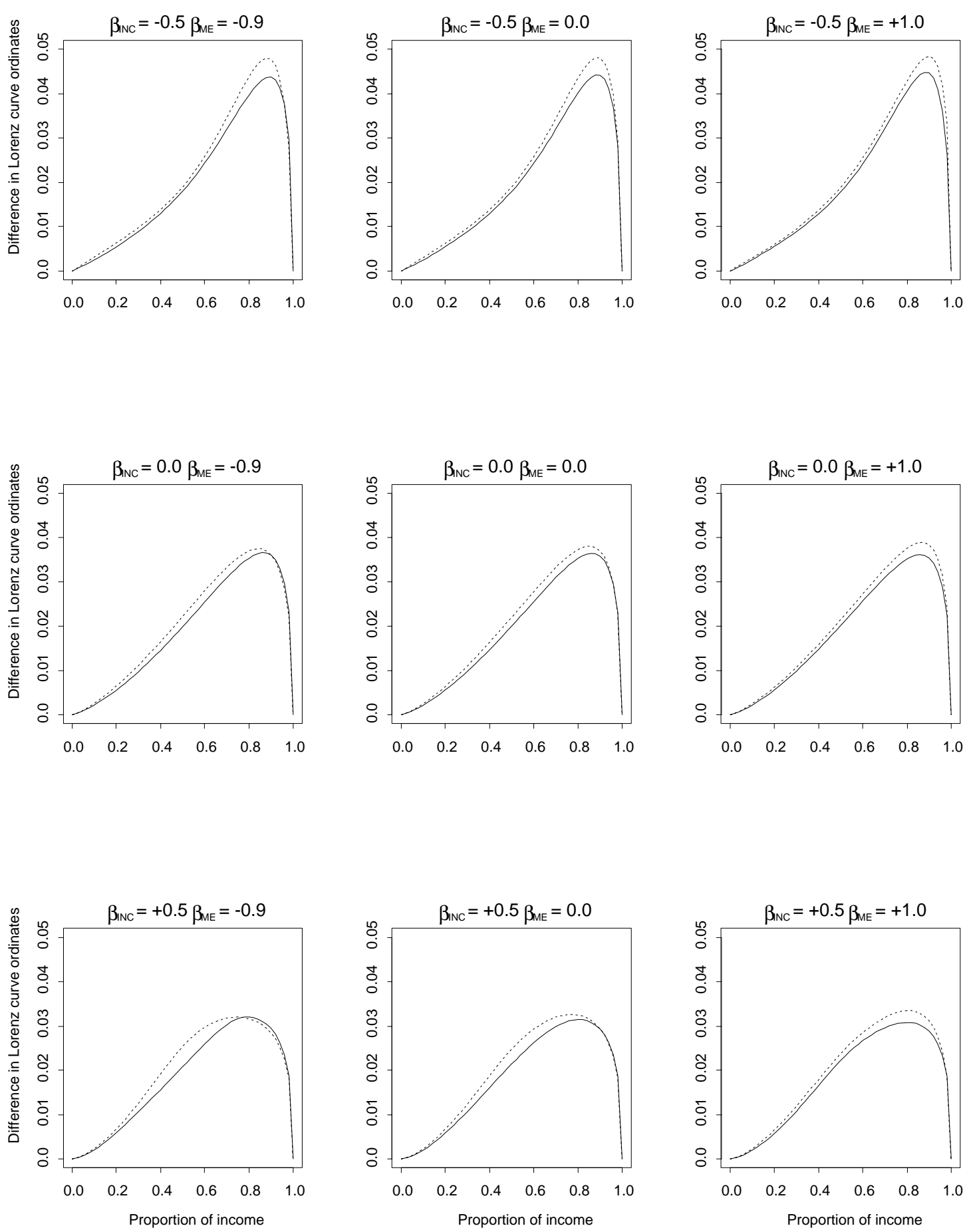
Figure 6: Exact (dashed) and approximate (solid) error contaminated Gini coefficeints expressed as ratios to error free values. Shape parameters: error free income $\beta_{I N C}$, measurement error $\beta_{M E}$.
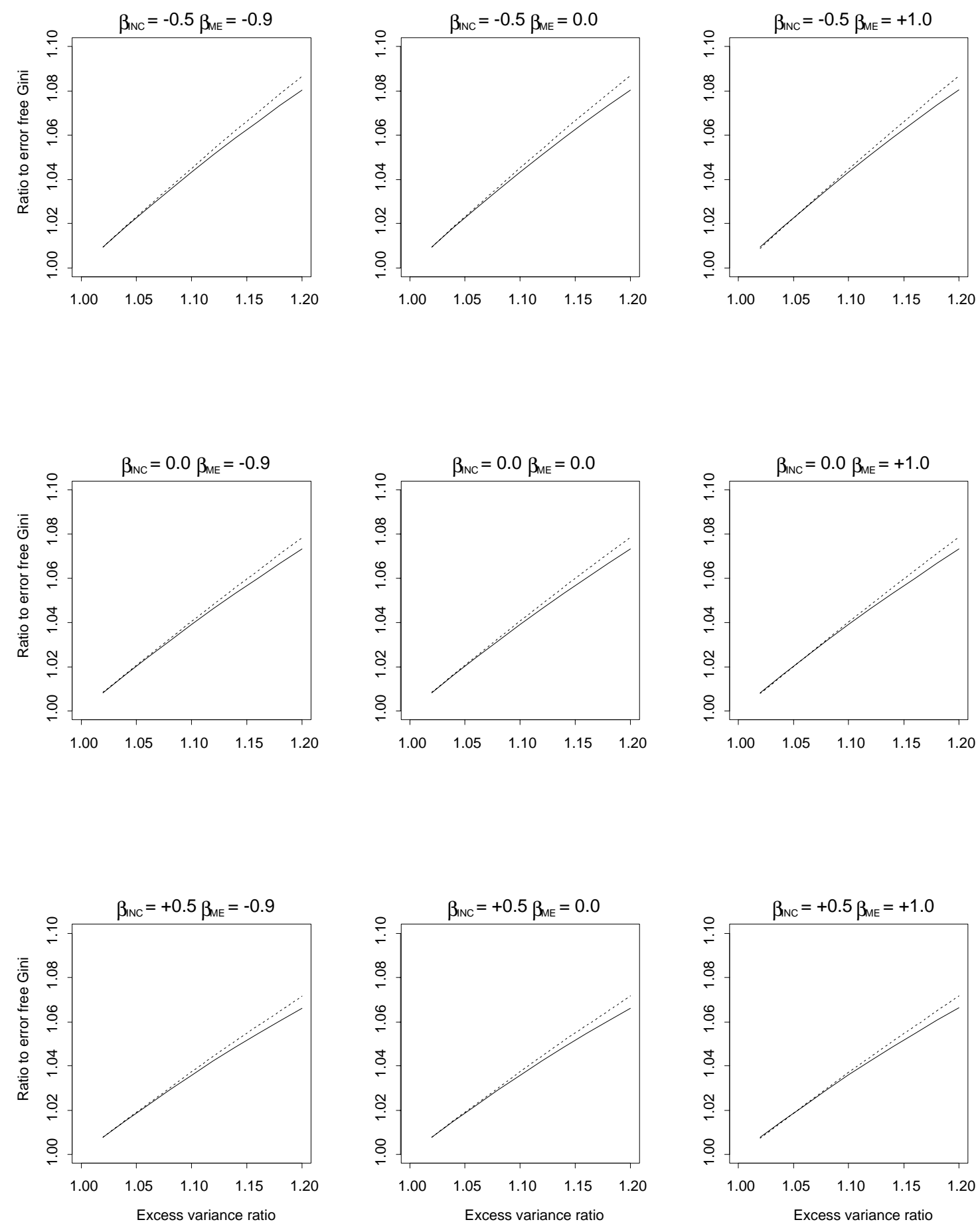


\section{ESTIMATION OF THE APPROXIMATE MEASUREMENT ERROR DISTORTION}

The approximations give insight into the relative sensitivity of different welfare measures to measurement error and the way in which sensitivity varies with the form of the income distribution. They also have more practical uses. To see this rewrite the generic form of the approximations as follows

$$
\mathcal{W}\left(F_{X}\right) \simeq \mathcal{W}\left(F_{Z}\right)-\sigma^{2} \mathcal{W}^{*}\left(F_{Z}\right)
$$

or equivalently, to the order of approximation considered, in multiplicative form as follows.

$$
\mathcal{W}\left(F_{X}\right) \simeq \mathcal{W}\left(F_{Z}\right) \exp \left(-\sigma^{2} \frac{\mathcal{W}^{*}\left(F_{Z}\right)}{\mathcal{W}\left(F_{Z}\right)}\right) .
$$

Since the welfare measures considered are all by construction non-negative, we might expect this multiplicative form to perform better, particularly when $\mathcal{W}\left(F_{Z}\right)$ is small.

Applying a welfare measure to error contaminated data produces an estimate of $\mathcal{W}\left(F_{Z}\right)$ but it is $\mathcal{W}\left(F_{X}\right)$ that is of interest. With an estimate of $\mathcal{W}^{*}\left(F_{Z}\right)$ it is possible to estimate the welfare measure for error free data, $\mathcal{W}\left(F_{X}\right)$, if an extraneous estimate of $\sigma^{2}$ is available. Even without such an estimate one may have a view of a plausible range of values for $\sigma^{2}$ in which case one can obtain an approximate interval estimate of the error free welfare measure.

In this Section we propose simple estimators of the "correction terms", $\mathcal{W}^{*}\left(F_{Z}\right)$. The accuracy of the approximations exact calculations of the previous Section suggest that knowledge of $\mathcal{W}^{*}\left(F_{Z}\right)$ will be highly informative, but it is possible that errors introduced by estimating the terms $\mathcal{W}^{*}\left(F_{Z}\right)$ will reduce the value of the procedure. To get an idea of whether this is likely to be the case we report a small Monte Carlo experiment focussing on the sampling distributions of estimates of the correction terms.

We propose to estimate each term $\mathcal{W}^{*}\left(F_{Z}\right)$ by applying the functional $\mathcal{W}^{*}$ to a nonparametric estimate of $F_{Z}$. In some cases it is possible to use the estimate $\mathcal{W}^{*}\left(\hat{F}_{Z}^{E D F}\right)$ where $\hat{F}_{Z}^{E D F}$ is the empirical distribution function $(\mathrm{EDF})$. In other cases (e.g. where $\mathcal{W}^{*}$ involves derivatives of $F_{Z}$ ) we propose using kernel estimators, in particular with Gaussian kernels because with this choice rather simple expressions for the welfare measure corrections arise. Since income data are usually heavily skewed we expect better performance applying the kernel method to log income data. The density estimators for (log income) data $\left\{y_{i}\right\}_{i=1}^{n}$ take the generic form

$$
\hat{f}_{Y}(y)=\frac{1}{n} \sum_{i=1}^{n} K_{h}\left(y-y_{i}\right)=\frac{1}{n} \sum_{i=1}^{n} \frac{1}{h} \phi\left(\frac{y-y_{i}}{h}\right)
$$

where $\phi$ is the standard normal density function, and $h$ is a bandwidth parameter.

5.1. Poverty indices with distribution independent poverty lines. First consider kernel based estimation of the poverty indices using the error contaminated income 
data. Let $R=\log Z$ have density function $f_{R}(r)$. The poverty indices in terms of the density of log income are

$$
P_{Z}(\theta, c)=\int_{-\infty}^{\log c}\left(1-\frac{\exp (r)}{c}\right)^{\theta} f_{R}(r) d r
$$

and replacing the unknown log income density by an estimator based on $n$ realised log income values, $\left\{r_{i}\right\}_{i=1}^{n}$, gives the estimator

$$
\hat{P}_{Z}(\theta, c)=\frac{1}{n} \sum_{i=1}^{n} \int_{-\infty}^{\log c}\left(1-\frac{\exp (r)}{c}\right)^{\theta} K_{h}\left(r-r_{i}\right) d r
$$

which for the three cases considered here take the following forms.

$$
\begin{gathered}
\hat{P}_{Z}(0, c)=\frac{1}{n} \sum_{i=1}^{n} \Phi\left(\frac{\log c-r_{i}}{h}\right) \\
\hat{P}_{Z}(1, c)=\hat{P}_{Z}(0, c)-\frac{1}{n c} \sum_{i=1}^{n} \exp \left(r_{i}+\frac{h^{2}}{2}\right) \Phi\left(\frac{\log c-\left(r_{i}+h^{2}\right)}{h}\right) \\
\hat{P}_{Z}(2, c)=2 \hat{P}_{Z}(1, c)-\hat{P}_{Z}(0, c)+\frac{1}{n c^{2}} \sum_{i=1}^{n} \exp \left(2\left(r_{i}+h^{2}\right)\right) \Phi\left(\frac{\log c-\left(r_{i}+2 h^{2}\right)}{h}\right)
\end{gathered}
$$

Now consider estimation of the measurement error correcting functionals $\mathcal{W}^{*}$ which in this section we denote by $C_{Z}(\theta, c)$. First consider the head count index which, for a poverty line drawn at $c$ has (see equation (18))

$$
\begin{aligned}
C_{Z}(0, c) & =\frac{1}{2}\left(c^{2} f_{Z}^{\prime}(c)+2 c f_{Z}(c)\right) \\
& =\frac{1}{2}\left(f_{R}(\log c)+f_{R}^{\prime}(\log c)\right)
\end{aligned}
$$

where $f_{R}$ is the density function of log income. This is replaced by a kernel density estimate to produce the estimated functional. The resulting estimate is as follows.

$$
\widehat{C}_{Z}(0, c)=\frac{1}{2 n} \sum_{i=1}^{n}\left(1-\frac{\log c-r_{i}}{h^{2}}\right) \frac{1}{h} \phi\left(\frac{\log c-r_{i}}{h}\right)
$$

The poverty index with $\theta=1$ has (see equation (19))

$$
\begin{aligned}
C_{Z}(1, c) & =\frac{1}{2}\left(c f_{Z}(c)\right) \\
& =\frac{1}{2} f_{R}(\log c)
\end{aligned}
$$

which we estimate by

$$
\widehat{C}_{Z}(1, c)=\frac{1}{2 n} \sum_{i=1}^{n} \frac{1}{h} \phi\left(\frac{\log c-r_{i}}{h}\right)
$$


The poverty index with $\theta=2$ has (see equation (21))

$$
C_{Z}(2, c)=P_{Z}(2, c)-2 P_{Z}(1, c)+P_{Z}(0, c)
$$

and combining the estimates of the three poverty indices gives

$$
\begin{aligned}
\widehat{C}_{Z}(2, c) & =\frac{1}{n c^{2}} \sum_{i=1}^{n} \int_{-\infty}^{\log c} \exp (2 r) K_{h}\left(r-r_{i}\right) d r \\
& =\frac{1}{n c^{2}} \sum_{i=1}^{n} \exp \left(2\left(r_{i}+h^{2}\right)\right) \Phi\left(\frac{\log c-\left(r_{i}+2 h^{2}\right)}{h}\right) .
\end{aligned}
$$

The poverty indices and $C_{Z}(2, c)$ can also be estimated directly by replacing the distribution function of $Z$ by the empirical distribution function (EDF) in their defining equations.

5.2. The Gini coefficient. The Gini coefficient is conveniently estimated using the relative mean difference form

$$
\widehat{G C}_{Z}=\frac{1}{2 \bar{z}} \frac{1}{n^{2}} \sum_{i=1}^{n} \sum_{j=1}^{n}\left|z_{i}-z_{j}\right|
$$

where $\bar{z}$ is sample mean income. This is an empirical distribution function (EDF) based method. The measurement error correction term prior to multiplication by $\sigma^{2}$ is as follows.

$$
A_{Z}=\frac{E\left[Z^{2} f_{Z}(Z)\right]}{E[Z]}
$$

Since $E\left[Z^{2} f_{Z}(Z)\right]=E\left[\exp (R) f_{R}(R)\right]$ where $R=\log (Z)$, the correction term can be estimated by the kernel method as ${ }^{12}$

$$
\begin{aligned}
\hat{A}_{Z} & =\frac{1}{\bar{z}} \int_{-\infty}^{\infty} \exp (r) \frac{1}{n^{2}} \sum_{i=1}^{n} \sum_{j=1}^{n} \frac{1}{h} \phi\left(\frac{r-r_{i}}{h}\right) \frac{1}{h} \phi\left(\frac{r-r_{j}}{h}\right) d r \\
& =\frac{1}{\bar{z}} \frac{1}{2 h \sqrt{\pi}} \frac{1}{n^{2}} \sum_{i=1}^{n} \sum_{j=1}^{n} \exp \left(-\left(\frac{r_{i}-r_{j}}{2 h}\right)^{2}+\frac{1}{2}\left(r_{i}+r_{j}\right)+\frac{h^{2}}{4}\right)
\end{aligned}
$$

\footnotetext{
${ }^{12}$ Alternatively one could use a single kernel to produce an estimate $\hat{f}_{R}(r)$ and then estimate $\hat{A}_{Z}$ as follows.

$$
\hat{A}_{Z}=\frac{1}{n \bar{z}} \sum_{i=1}^{n} \exp \left(r_{i}\right) \hat{f}_{R}\left(r_{i}\right)
$$
}


Table 3: Monte Carlo Estimates of means and standard deviations of estimators of welfare indexes and measurement error corrections

\begin{tabular}{|c|c|c|c|c|c|c|c|c|c|}
\hline \multirow{3}{*}{$\begin{array}{l}\text { Index or } \\
\text { Correction }\end{array}$} & \multirow[b]{3}{*}{ True } & \multicolumn{4}{|c|}{ Sample size 200} & \multicolumn{4}{|c|}{ Sample size 500} \\
\hline & & \multicolumn{2}{|c|}{ Kernel } & \multicolumn{2}{|c|}{ EDF } & \multicolumn{2}{|c|}{ Kernel } & \multicolumn{2}{|c|}{ EDF } \\
\hline & & Mean & S.D. & Mean & S.D. & Mean & S.D. & Mean & S.D. \\
\hline$\hat{P}_{Z}(0, c)$ & .159 & .170 & .022 & .159 & .025 & .167 & .016 & .160 & .016 \\
\hline$\widehat{C}_{Z}(0, c)$ & .242 & .229 & .044 & $\begin{array}{lll}- & - \\
\end{array}$ & - & .232 & .034 & $\begin{array}{ll}- & \text { r }\end{array}$ & - \\
\hline$\hat{P}_{Z}(1, c)$ & .057 & .064 & .011 & .057 & .011 & .062 & .007 & .057 & .007 \\
\hline$\widehat{C}_{Z}(1, c)$ & .121 & .120 & .014 & - & $\begin{array}{ll}- & \text { r }\end{array}$ & .121 & .010 & - & - \\
\hline$\hat{P}_{Z}(2, c)$ & .028 & .033 & .007 & .028 & .007 & .032 & .005 & .029 & .004 \\
\hline$\widehat{C}_{Z}(2, c)$ & .074 & .076 & .009 & .074 & .014 & .076 & .007 & .074 & .009 \\
\hline $\bar{G} \bar{C}_{Z}$ & .521 & - & - & .515 & .028 & - & - & .519 & .019 \\
\hline$\hat{A}_{Z}$ & .220 & .220 & .020 & - & - & .218 & .014 & - & - \\
\hline
\end{tabular}

5.3. Estimator performance. Table 3 shows the results of a 1000 replication Monte Carlo experiment using a standard log normal distribution for $Z$, conducted to assess the performance of the proposed estimators of the poverty indices $\left(\hat{P}_{Z}(\theta, c), \theta \in\{0,1,2\}\right)$ and measurement error correction terms $\left(\widehat{C}_{Z}(\theta, c)\right)$ and of the Gini coefficient estimator $\widehat{G C}_{Z}$ and the estimator of its correction term $\hat{A}_{Z}$. The bandwidth was chosen at each replication using Silverman's "rule of thumb" (Silverman (1986)) which gave very similar results to those obtained with more computationally demanding cross-validation ${ }^{13}$. Means and standard deviations (SD) are reported for each estimator. The experiments were run with a sample size at each replication, $n$, equal to 200 and 500. The column headed "True" gives exact values, calculated using the methods of the previous Section.

There is some slight upward bias in the kernel based estimates of the poverty indices which is absent for the EDF based estimates. However there seems to be little bias in the kernel based estimates of the correction terms for $\theta \geq 1$. The estimates of the Gini coefficient and its correction display little sign of bias.

This small experiment suggests that in samples of the sort of size frequently encountered in inequality and poverty measurement quite accurate estimation of the proposed measurement error corrections is possible. The accuracy of the corrections documented in the previous Sections suggests that there is scope for useful application of estimated approximate measurement error corrections in practical welfare measurement exercises. The next Section illustrates such an application.

\footnotetext{
${ }^{13}$ Cross validation was used in the application in Section 6.
} 


\section{AN APPLICATION TO REGIONAL POVERTY AND INEQUALITY COMPARISONS IN INDONESIA}

This Section illustrates the use of these procedures in a sensitivity analysis of the sort that could be conducted when using regional welfare measures to inform the targeting of poverty interventions. Targeting might be based on observed differences in welfare measures across regions. But these differences could arise because of differences in amounts of measurement error. This could be an important issue when comparing measures across urban and rural areas. Accurate measurement may be more difficult to achieve in the country where there are likely more non-market transactions, consumption of own production and associated imputation of prices.

In this illustrative example the data employed are monthly per capita household expenditures (1993 Rupiah per person per month (Rppm)) reported in the 1993 Indonesian household survey SUSENAS in urban and rural areas in four provinces of Indonesia with poverty lines set here, for illustration only, at 20,000 rupiah per person per month in each $\operatorname{area}^{14}$.

We calculate three welfare measures for each area, estimate the measurement error correction terms, and then apply these for a range of values of measurement error log variance. We focus on the Gini coefficient, $G C_{Z}$ and on the head count index, $P_{Z}(0, c)$ and the poverty gap index, $P_{Z}(1, c)$, both with poverty line $c=20,000 \mathrm{Rppm}$. For log measurement error variance $\sigma^{2}$ the approximately corrected welfare measures are as follows.

$$
\begin{aligned}
\hat{P}_{X}(0, c) & =\hat{P}_{Z}(0, c)-\frac{\sigma^{2}}{2}\left(c^{2} \hat{f}_{Z}^{\prime}(c)+2 c \hat{f}_{Z}(c)\right) \\
\hat{P}_{X}(1, c) & =\hat{P}_{Z}(1, c)-\frac{\sigma^{2}}{2} c \hat{f}_{Z}(c) \\
\widehat{G C}_{X} & =\widehat{G C}_{Z}-\sigma^{2} \frac{\hat{E}\left[Z^{2} f_{Z}(Z)\right]}{\hat{E}[Z]}
\end{aligned}
$$

Here $f_{Z}$ is the density of (error contaminated) expenditure per head, $Z, f_{Z}^{\prime}$ is its derivative, and $\hat{f}_{Z}$ and $\hat{f}_{Z}^{\prime}$ are nonparametric estimates of the density and its derivative.

We ask: could plausibly different amounts of measurement error across the areas be responsible for the differences in the welfare measures that we see when they are computed using the raw, error contaminated, survey data?

Table 4 shows the results of the calculations ${ }^{15}$. The rows labelled $\sigma^{2}=0$ show the welfare measures before correction for measurement error. Poverty measures are higher, and Gini coefficients lower, in rural areas than in urban areas. The Table suggests that

\footnotetext{
${ }^{14}$ SUSENAS is the "Survei Sosial Ekonomi Nasional" described in Surbaki (1995). An analysis of poverty incidence using the survey can be found in Bidani and Ravallion (1993). Sample sizes (households: urban then rural) are as follows: C. Java: 2366, 4337; Lampung: 701, 1319; W. Nusa Tenggara: 708, 1342; E. Nusa Tenggara: 492, 1191.

${ }^{15}$ The poverty indexes and the Gini coefficient have been calculated using an EDF estimator. The corrections have been calculated using kernel methods with bandwidth chosen using the Fast Fourier approximation to the cross validation method as proposed by Silverman (1986).
} 
Table 4: Estimated poverty indexes and Gini coefficients for error free income at alternative values of measurement error log variance

\begin{tabular}{|c|c|c|c|c|c|c|c|}
\hline \multirow[b]{3}{*}{ Province } & \multirow[b]{3}{*}{$\sigma^{2}$} & \multicolumn{3}{|c|}{ URBAN AREAS } & \multicolumn{3}{|c|}{ RURAL AREAS } \\
\hline & & \multicolumn{2}{|c|}{ Poverty indexes } & Gini & \multicolumn{2}{|c|}{ Poverty Indexes } & Gini \\
\hline & & $\hat{P}_{X}(0, c)$ & $\hat{P}_{X}(1, c)$ & $\widetilde{G C_{X}}$ & $\hat{P}_{X}(0, c)$ & $\hat{P}_{X}(1, c)$ & $\widetilde{G C_{X}}$ \\
\hline \multirow{4}{*}{$\begin{array}{l}\text { Central. } \\
\text { Java }\end{array}$} & .00 & .055 & .007 & .313 & .236 & .041 & .273 \\
\hline & .02 & .043 & .005 & .304 & .216 & .033 & .262 \\
\hline & .04 & .035 & .003 & .295 & .197 & .027 & .252 \\
\hline & .06 & .028 & .002 & .286 & .180 & .022 & .241 \\
\hline \multirow[t]{4}{*}{ Lampung } & .00 & .054 & .007 & .303 & .251 & .044 & .256 \\
\hline & .02 & .044 & .005 & .294 & .236 & .037 & .245 \\
\hline & .04 & .036 & .004 & .285 & .222 & .031 & .234 \\
\hline & .06 & .030 & .003 & .276 & .209 & .025 & .224 \\
\hline W. Nusa & .00 & .075 & .011 & .316 & .247 & .040 & .254 \\
\hline \multirow[t]{3}{*}{ Tenggara } & .02 & .065 & .008 & .307 & .228 & .032 & .242 \\
\hline & .04 & .057 & .006 & .299 & .211 & .026 & .231 \\
\hline & .06 & .049 & .004 & .291 & .195 & .021 & .221 \\
\hline E. Nusa & .00 & .063 & .007 & .344 & .267 & .052 & .230 \\
\hline \multirow[t]{3}{*}{ Tenggara } & .02 & .054 & .005 & .336 & .241 & .044 & .217 \\
\hline & .04 & .047 & .004 & .328 & .218 & .037 & .204 \\
\hline & .06 & .040 & .002 & .321 & .198 & .031 & .193 \\
\hline
\end{tabular}

within each province rural and urban sectors appear to be very different, but that the same sectors across the provinces are rather similar.

The remaining rows show estimates of the poverty indexes and Gini coefficients after correction $^{16}$ for varying amounts of measurement error with $\sigma^{2}$ denoting the conjectured variance of $\log$ measurement error. The variance of log expenditure per head is between 0.2 and 0.3 for the areas considered here, so measurement error with log variance in the range 0 to 0.06 used in Table 4 corresponds to an excess log variance ratio in the range 1 to 1.4 .

These results suggest that the differences in measured poverty and inequality across rural and urban areas cannot plausibly be explained by differences in measurement error. Even large amounts of measurement error in rural areas suggest error free poverty indices far above, and Gini coefficients far below, the measured values for urban areas. However, it is clear that differences in the incidence of measurement error of the sorts of magnitude considered here could seriously disturb the view of regional differences in inequality when comparisons are made within rural or urban areas.

\footnotetext{
${ }^{16}$ The multiplicative form (22) of the correction has been used here.
} 


\section{CONCLUding REMARKS}

Measurement error is an ever-present, generally significant, but usually neglected, feature of survey based income and expenditure data. Since it is usually variance-increasing, measurement error can be expected to have a significant impact on inequality and poverty measurement. This paper has provided approximations to the effect of measurement error on a variety of welfare measures, both inequality measures and poverty indices. We have shown how these can be used to investigate the sensitivity of analysis of regional poverty and inequality measures to differential incidence of measurement error.

The approximations show how welfare measures for error free income distributions deviate from welfare measures for error contaminated income distributions, the deviation to a first approximation being independent of the shape of the measurement error distribution and determined by the measurement error log variance and estimable functionals of the error contaminated income distribution. The approximations are quite accurate for a wide variety of true income and measurement error distributions even when there are moderate amounts of measurement error. We have proposed methods for estimating these functionals and investigated their performance. They can be estimated accurately with samples of the size typically encountered in applied welfare measurement.

Our approximations are valid, and seem accurate, for a wide range of error free income and independent measurement error distributions, but there may arise cases in which the independence assumption is hard to justify. With a particular model for dependence progress along similar lines could be made, but it may be difficult to formulate and identify a suitable model. One would need validation data to make real headway but with that one could develop exact inferential procedures and the methods of this paper would not be needed. The tool developed here allows assessment of the potential impact of measurement error under independence, the leading case of interest, when, as is normally the case, all that one has to work with is the measurement error contaminated survey data.

\section{ACKNOWLEDGMENTS}

We are grateful to Martin Browning, Frank Cowell, to two referees and an editor of the Journal for helpful comments. Andrew Chesher and Christian Schluter received support under ESRC grants, respectively: R008237386 and R000222650. 


\section{REFERENCES}

Atkinson, A.B. (1970) "On the Measurement of Inequality", Journal of Economic Theory, 2, 244-263.

Bidani, B. And Ravallion, M. (1993) "A New Regional Poverty Profile for Indonesia" Bulletin of Indonesian Economic Studies, 29, 37-68.

Box, G.E.P., AND TiaO, G.C. (1973) Bayesian Inference in Statistical Analysis, New York: Wiley Classics.

Chesher, A.D. (1991) "The Effect of Measurement Error", Biometrika, 78, 451 - 462.

Chesher, A.D. (1997) "Non-normal Variation and Regression to the Mean", Statistical Methods in Medical Research, 6, 147-166.

Chesher, A.D. Dumangane, M.B.G. And Smith, R.J. (2001) "Duration Response Measurement Error", forthcoming, Journal of Econometrics.

Chesher, A.D., And Schluter, C. (1999) "Welfare Measurement and Measurement Error" University of Bristol Department of Economics Discussion Paper No. 98/463.

Cowell, F. A. (1980) "On the Structure of Additive Inequality Measures", Review of Economic Studies, 47, 521-531.

Cowell, F. A. (2000) "Measurement of Inequality", in Handbook of Income Distribution, ed., Atkinson, A. B. and F. Bourguignon, Amsterdam: North Holland.

Cowell, F. A. AND Victoria-Feser, M.-P. (1996) "Robustness Properties of Inequality Measures", Econometrica, 64, 77-101.

DAvidson, R. AND Duclos, J.-Y. (1997) "Statistical Inference for the Measurement of the Incidence of Taxes and Transfers", Econometrica, 65, 1453-1465.

Foster, J. E. And Greer, J. And Thorbecke, E. (1984) "A Class of Decomposable Poverty Measures", Econometrica, 52, 761-776.

Israelsen, D. AND MCDonald, J.B. AND NeWEy, W.K. (1984) "The Impact of Measurement Error on the Distribution of Income", Advances in Econometrics, JAI Press. Ravallion, M. (1994), "Poverty Rankings Using Noisy Data on Living Standards", Economics Letters, 45, 481-485.

SAPosnik, R. (1981) "Rank Dominance in Income Distribution", Public Choice, 36, 147-151.

Saposnik, R. (1983) "On Evaluating Income Distributions: Rank Dominance", Public Choice, 40, 329-336.

SEIDL, C. (1988) "Poverty Measurement: A Survey" in Welfare and Efficiency in Public Economics, ed., Boes, D. and Rose, M. and Seidl, C., Berlin:Springer-Verlag.

Shorrocks, A. F. (1983) "Ranking Income Distribution", Economica, 50, 3-17.

Silverman, B.W. (1986) Density Estimation for Statistics and Data Analysis, London: Chapman and Hall.

Surbaki, P. (1995) Indonesia's National Socio-Economic Survey, Jakarta: Central Bureau of Statistics.

van Praag, B. Hagenaars, A. and van Eck, W. (1983) "The Influence of Classification and Observation Error on the Measurement of Income Inequality", Econometrica, 51 1093-1108. 